\title{
Innovación y conocimiento libre: cuestiones morales y políticas
}

\section{Innovation and open knowledge: moral and political issues}

\author{
JAVIER DE LA CUEVA GONZÁlEZ-CoterA
}

Universidad Complutense de Madrid

RESUMEN. En el presente trabajo se parte de que la innovación requiere transmisión de información. En la actualidad, el contenido de la transmisión de información se halla sometido a una regulación legal, habitualmente la propiedad intelectual, donde los agentes intervinientes y su requisito de sistema de permisos dificultan la transmisión libre. Para solucionar esta problemática, se están promoviendo dos tipos de soluciones: el primero mediante código legal y el segundo mediante código informático. Estas soluciones, que se fundamentan en razones morales, tienen consecuencias políticas y determinan el modelo de sociedad que podemos construir mediante el conocimiento transmitido.

Palabras clave: Innovación, conocimiento libre, democracia, transparencia, propiedad intelectual
ABSTRACT. In this paper we assume that innovation requires transmission of information. The content of the transmitted information currently is subject to legal regulation, typically intellectual property, where the agents and the requirement of a needed consent hinder free transmission. To solve this problem, two type of solutions are being implemented: first through legal code and second through computer code. These solutions, rooted on ethical foundations, have political consequences and determine the kind of society we can build using the transmitted knowledge.

Key words: Innovation, open knowledge, democracy, transparency, intellectual property

\section{Introducción}

Para que exista innovación, debe existir transmisión de conocimiento. En el actual período histórico, la transmisión de las obras literarias, artísticas y científicas, base del conocimiento, se halla regulada por una legislación de propiedad intelectual muy homogeneizada globalmente, lo que se logra mediante diversos tratados internacionales que marcan los rasgos generales del contenido de las normas, que luego los diversos Estados desarrollan para ser aplicadas en su territorio, y que hacen primar los derechos privados frente a los públicos. Es 
característico de esta regulación un sistema de permisos para cada uno de los usos concretos de una obra, lo que exige que para utilizarla legalmente se requiera bien un consentimiento previo para la utilización específica que se quiera realizar de la obra, consentimiento concedido al usuario por el titular del derecho, bien que el usuario pueda ampararse en un límite del derecho del titular específicamente contemplado en la ley, bien que la obra se halle en dominio público, lo que analizaremos sintéticamente en este artículo.

Partiendo de esta situación y utilizando el modelo matemático de la comunicación de Shannon (1948), analizaremos cómo ciertos titulares de derechos de propiedad intelectual han litigado judicialmente en nuestra jurisdicción contra todos y cada uno de los sujetos que intervienen en el citado modelo en el entorno de las Tecnologías de la Información y Comunicación (TIC), operando así como reguladores de la transmisión del conocimiento.

Señalaremos cómo, frente a este contexto y para facilitar el conocimiento libre, diversos actores han aportado soluciones que podemos agrupar en legales y tecnológicas. Las soluciones legales comenzaron mediante la práctica de estandarizar licencias de propiedad intelectual para permitir la reutilización total o parcial de las obras, su distribución, difusión y transformación (las $l i$ cencias libres). Posteriormente, y ante la existencia de información no sometida a propiedad intelectual pero sí a otros sistemas regulatorios (fundamentalmente la reutilización de la información del sector público), surgió la necesidad de concretar lo que se entiende por libre/abierto, surgiendo como herramienta conceptual la open definition, con cuya iniciativa se pretende diferenciar entre la diversa granularidad de los sistemas de reutilización. En lo que se refiere a las soluciones tecnológicas, el desarrollo de código informático bajo licencias libres ha facilitado la transmisión de información tanto haciendo inaplicables por obsoletas las regulaciones legales como tendiendo arquitecturas.

A continuación trataremos las cuestiones morales y políticas que se derivan del conocimiento libre. Partiendo de los derechos a la educación y a la igualdad y su incidencia en la circulación de las élites, señalaremos la ilegitimidad que se introduce en los sistemas democráticos a través de la intervención en la calidad de la información, lo que se sintetiza en la perversa relación de los elementos de la tríada dinero, información y política: dinero para hacer política e información, información para hacer dinero y política, política para hacer dinero e información (Ferrajoli, 2010a, 271, y 2011, 53). Dado que las políticas públicas (policy) sobre conocimiento e innovación son una subclase de las políticas generales, podremos presumir que si la información utilizada para tomar decisiones políticas sobre aspectos generales está sujeta a la voluntad de grupos que regulan su transmisibilidad en favor de intereses privados, tam- 
bién lo estarán las informaciones sobre las que se deban apoyar el conocimiento y la innovación ${ }^{1}$.

Posteriormente, tras apuntar que el conocimiento libre es consustancial a las normas jurídicas y resoluciones judiciales, encontraremos cómo la incidencia de este fenómeno de pérdida de la calidad de la información alcanza a los tres elementos sustrato de una sociedad democrática: los valores encarnados por los derechos fundamentales, la ordenación del debate y la fijación del orden democrático. Sobre los derechos fundamentales, y de acuerdo con la necesidad de reflexionar a fondo sobre las componentes básicas de una sociedad [...] democrática cuyo contexto es [e]l tránsito de la ciudadanía urbana e industrial [...] a la infociudadanía (Echeverría, 2003, 90), analizaremos cómo existen derechos fundamentales con sustrato informacional incorporado mientras que otros no participan de esta característica. Sobre el aspecto organizacional, estudiaremos cómo la permanente búsqueda de una sociedad abierta democrática dependerá no sólo de cómo se produzca la transmisión del conocimiento sino del retorno de la información entre los diversos agentes y los órganos estatales, donde encontraremos un derecho de participación de naturaleza informacional que se manifiesta mediante el derecho a saber y mantiene relaciones con la existencia de la corrupción y la necesidad de transparencia, en definitiva, el orden democrático. La conclusión final a la que llegaremos es que el conocimiento libre, lejos de tratarse únicamente de una cuestión de propiedad intelectual, es una forma de promover innovación y de ejercer políticas públicas para sociedades más democráticas.

\section{Dificultades para el desarrollo del conocimiento libre}

Nos hallamos en un mundo informacional donde cualquier uso de una obra objeto de propiedad intelectual se halla prohibido salvo (i) que se obtenga un permiso del titular de los derechos, (ii) que nos hallemos realizando una actividad englobada dentro de un límite de las leyes que rigen la propiedad intelectual o (iii) que la obra se halle en dominio público por transcurso del tiempo desde su creación. Aunque parezca obvio, merece la pena señalar que si bien nuestra regulación legal exige que para que nazcan los derechos de propiedad intelectual debe existir una obra que sea una creación original literaria, artística o científica ${ }^{2}$, en

\footnotetext{
${ }^{1}$ Sobre el concepto de innovación, cfr. Gurrutxaga y Echeverría (2012, 125-129).

${ }^{2}$ Artículo 10.1 del Real Decreto Legislativo 1/1996, de 12 de abril, por el que se aprueba el texto refundido de la Ley de Propiedad Intelectual, regularizando, aclarando y armonizando las disposiciones legales vigentes sobre la materia (TRLPI). Boletín Oficial del Estado número 97 de 22 de abril de 1996. Vid. Bercovitz (2007a).
} 
la práctica toda información es susceptible de ser objeto de litigio, siendo un campo en el que la introducción de cualquier tecnología novedosa ha sido objeto de controversia judicial por parte de los titulares de los derechos. Esta litigiosidad tiene efectos negativos en el desarrollo de la tecnología, siendo más evidente en el sistema de patentes (Bessen y Meurer, 2008; Vaikhari, 2008; McCurdy, 2009; Bessen, Meurer y Ford, 2011; Tucker, 2011) y, por tanto, en la innovación derivada de lo que denominamos propiedad industrial ${ }^{3}$.

Esta regulación legal es muy homogénea globalmente y su estructura, sintéticamente, se compone de una parte pública y una privada. En la parte pública, hallamos los tratados internacionales firmados en sede de la Organización Mundial de la Propiedad Intelectual (OMPI), la agencia de las Naciones Unidas dedicada al uso de la propiedad intelectual (patentes, derecho de autor, marcas, diseños -dibujos y modelos-, etc.) como medio de estimular la innovación y la creatividad ${ }^{4}$. Los tratados de la OMPI una vez firmados por los Estados son incorporados a sus legislaciones nacionales y, además, en nuestro caso, la Unión Europea desarrolla unas Directivas ${ }^{5}$ (acordes con los tratados) que posteriormente son objeto de transposición por los Estados miembros. Sin embargo, la OMPI carecía de un mecanismo coactivo para obligar a los países a adoptar las leyes del copyright (Smiers, 2008, 58 ), por lo que se impuso una parte privada mediante las negociaciones multilaterales de la Ronda Uruguay (1986-1994) que culminaron el 15 de abril de 1994 con la firma en Marraquech por representantes de la Unión Europea y de sus Estados miembros del Acta final del Acuerdo por el que se crea la Organización Mundial del Comercio (OMC). Como Anexo 1C al acuerdo de creación de la $\mathrm{OMC}$ se firmaron los «Aspectos de los derechos de propiedad intelectual relacionados con el comercio» (ADPIC) en cuyo preámbulo literalmente se establece que se reconoce que los derechos de propiedad intelectual son derechos privados siendo los objetivos fundamentales de politica general pública de los sistemas nacionales la protección de los derechos de propiedad intelectual, con inclusión de los objetivos en materia de desarrollo y tecnología. De esta manera y mediante una estructura piramidal se logra la homogeneización de un campo en el que operan los intereses privados de

\footnotetext{
${ }^{3}$ En otros sistemas legales, la propiedad industrial se incluye dentro del concepto de propiedad intelectual. Lo relevante es que ambas son propiedades especiales que nacen de formalizaciones de ideas.

${ }^{4}$ Vid. <http://www.wipo.int/about-wipo/es/>. Fecha de consulta: 29 de diciembre de 2012.

${ }^{5}$ Para una consulta exhaustiva de la normativa europea aplicable, ver la web de la Unión Europea sobre propiedad intelectual: <http://europa.eu/legislation_summaries/internal_market/businesses/intellectual_property/index_es.htm>. Fecha de consulta: 29 de diciembre de 2012.
} 
los titulares de los derechos y se promueven políticas públicas que les benefician ${ }^{6}$, en un mercado muy concentrado ${ }^{7}$.

Ahora bien, estamos hablando de información, un bien del que tanto Hess y Ostrom como Posner nos recuerdan que se trata de lo que los economistas clásicos categorizaron como un «bien público»: un bien al alcance de todos donde el uso realizado por una persona no aminora el uso realizado por otra (Hess y Ostrom, 2007, 8) y [l] a información, definida ampliamente para incluir los bienes simbólicos y expresivos, es un ejemplo de lo que llaman los economistas "bien público". Un bien público es un bien que puede consumirse sin reducir el consumo del mismo por parte de cualquier otra persona (Posner, 2007, 82). Si bien no podemos afirmar que Posner esté pensando en las Tecnologías de la Información y Comunicación (TIC), es común a ambas posturas que la imposibilidad de agotamiento del bien impide su escasez y por tanto la célebre ley de la oferta y demanda señalaría que al existir una oferta infinita, el precio habría de tender hacia prácticamente cero con lo que, sin duda, nos hallamos con un sistema que no es propicio para los intereses de los titulares de los derechos de propiedad intelectual.

Para mantener los precios, los titulares de los derechos han desarrollado una serie de estrategias ${ }^{8}$. Hemos señalado tres requisitos para poder utilizar obras de propiedad intelectual: tener el permiso de los titulares de los derechos, ejercitar un límite legal del derecho o que la obra se halle en dominio público. Pues bien, los titulares de las grandes carteras de derechos han presionado (y siguen presionando) a los gobiernos estatales para el desarrollo de legislaciones en las que la utilización de una obra sin permiso tenga castigos cada vez mayores, se reduzcan

${ }^{6}$ Sobre la operatividad entre intereses privados e intereses públicos, ver, con carácter general, Capella (2008, 307-312), Ferrajoli (2011, 52-58) y, específicamente con respecto a la propiedad intelectual, Drahos y Braithwaite (2002) y Sell (2003).

${ }^{7}$ Sobre la concentración en el ámbito de las publicaciones académicas, vid. Lafuente y Alonso $(2011,194)$ y en el ámbito de los beneficiarios de los derechos de propiedad intelectual, el informe de la Comisión Nacional de la Competencia es esclarecedor: conviene tener presente el caso de la SGAE, donde unos 600 titulares (un 1,7\% del total de titulares beneficiarios) concentran el 75\% de lo que se reparte (CNC, 2009, 26-27).

${ }^{8}$ Para un estudio profundo de las prácticas monopolísticas de las entidades de gestión nacionales, ver los informes de los órganos oficiales CNC (2009) y AEVAL (2008). La Comisión Nacional de la Competencia afirma literalmente que no es del todo descartable conforme a la jurisprudencia comunitaria que la Comisión Europea pueda llegar a considerar que España es responsable de una infracción del artículo 86 del Tratado CE (actual art. 106 del Tratado de Funcionamiento de la Unión Europea) [...] siendo así que el artículo 82 TCE (actual art. 102 del TFUE) prohibe a las empresas la explotación abusiva de su posición dominante en el mercado. $(2009,86)$. Ninguno de los gobiernos existentes desde entonces (PSOE y PP) ha hecho nada al respecto tras el informe de la CNC. 
al máximo los límites de los derechos y se extienda el tiempo ${ }^{9}$ hasta que una obra entre en dominio público ${ }^{10}$. La excusa es la de promover mercados de innovación y de creatividad, excusa que no se sustenta en prueba alguna más que en declaraciones de los interesados y que más bien parece haber logrado eliminar la diversidad local imponiendo la hegemonía de las corporaciones del entretenimiento (Smiers, 2006, 147-159), cumpliéndose de esta manera lo anunciado por Horkheimer y Adorno en 1944 (2009, 166): la técnica de la industria cultural ha llevado sólo a la estandarización y producción en serie y ha sacrificado aquello por lo cual la lógica de la obra se diferenciaba de la lógica del sistema social. No cabe sostener que cuanto más punitivo, ilimitado y perpetuo sea un sistema de propiedad intelectual, mejor calidad e innovación generará puesto que, si así fuera, no se entendería cómo se ha producido toda la cultura anterior a la invención de esta figura jurídica ni tendría explicación la obra documental regulada por propiedad intelectual más relevante de los últimos tiempos, los Request for Comments ${ }^{11}$, que además de ser la base y fundamento de los protocolos de internet, es de libre copia, difusión, distribución y transformación ${ }^{12}$. En síntesis: ni Aristóteles ni internet tendrían posible explicación. Hay mucha vida más allá de la propiedad intelectual punitiva, ilimitada y perpetua.

Pero la intervención de los partidarios de este modelo de propiedad intelectual no se limita a la presión sobre los gobiernos para la modificación de las normas sino que realizan una actividad incesante en los foros procesales. Si tomamos como mapa conceptual el modelo matemático de la comunicación de Shannon (1948), encontramos que la industria del entretenimiento y algunas entidades de gestión han litigado en España contra todos y cada uno de los eslabones de la cadena de comunicación. Nótese que no estamos defendiendo la validez del modelo de Shannon ${ }^{13}$, sino que lo utilizamos como herramienta descriptiva para ordenar lo que aparentan ser procedimientos aislados y sin ninguna relación entre sí. El modelo de Shannon supone la existencia de los si-

${ }^{9}$ La pretensión de prolongar a perpetuidad los derechos de autor viene de lejos. Patterson $(1968,163)$ señala que el primer caso fue en 1743, Millar vs. Kinkaid, ante la corte escocesa. En el mismo sentido, Rose (1993, 67-91), quien asimismo detalla los litigios habidos en el siglo XVIII.

${ }^{10}$ Vid. Lessig (2005).

${ }^{11}$ Vid. <http://www.rfc-editor.org/rfc.html>. Fecha de consulta: 29 de diciembre de 2012.

${ }^{12}$ Para las condiciones de propiedad intelectual aplicables a los RFCs, ver IETF Trust Copyright Policy and Trust Legal Provisions (TLP). Frequently Asked Questions. June 22, 2010. $<$ http://trustee.ietf.org/docs/Copyright-FAQ-2010-6-22.pdf $>$ Fecha de consulta: 29 de diciembre de 2012.

${ }^{13} \mathrm{Ni}$ tampoco debemos confundir el modelo matemático de la comunicación con las ecuaciones de Shannon. Para una explicación de las ecuaciones, ver Aleksander (2004). 
guientes elementos: (1) un emisor que (2) codifica (3) un mensaje que transmite a través de (4) un canal, el cual llega a (5) un receptor que lo (6) descodifica; la acción se realiza en un entorno de (7) ruido. Una muestra de los litigios interpuestos es la siguiente ${ }^{14}$ :

- (1) y (5): Emisor y receptor. La industria ha ejercitado la acusación penal contra los usuarios de las redes de transmisión de archivos entre pares, redes $\mathrm{p} 2 \mathrm{p}^{15}$. - (2) y (6): Codificación y descodificación del mensaje. En este caso, la acusación penal de la industria se ha ejercitado tanto contra los usuarios como los establecimientos que venden modchips, unos dispositivos tecnológicos para que las consolas de videojuegos puedan utilizar juegos de otros proveedores y también grabados por los usuarios ${ }^{16}$.

- (3): Mensaje. La Sociedad General de Autores y Editores (SGAE) interpone habitualmente procedimientos de reclamación de cantidad contra los establecimientos con independencia de que se utilice el repertorio cuya gestión tiene encomendada ${ }^{17}$. Igualmente, el Centro Español de Derechos Reprográficos (CEDRO) ha interpuesto demandas contra dos universidades por utilización de lo que considera su repertorio ${ }^{18}$.

- (4): Canal. Sobre los soportes de los mensajes (cualquier aparato, equipo o material susceptible de hacer copias) sufrimos un gravamen por copia privada cuya regulación nacional anterior fue declarada ilegal por el Tribunal

${ }^{14}$ La persecución en los foros procesales no sólo se produce en nuestra jurisdicción sino que este modelo se reproduce en menor o mayor medida en múltiples jurisdicciones.

${ }^{15}$ La sentencia de 18 de febrero de 2008 de la Audiencia Provincial de Cantabria desestimó la acusación penal de la Asociación Fonográfica y Videográfica Española (AFYVE) (hoy denominada Promusicae -patronal de la industria fonográfica-), Columbia Tristar Home Entertainment, Walt Disney Company Ibérica y la Asociación Española de Distribuidores y Editores de Software de Entretenimiento (ADESE) contra un usuario de redes p2p al que se le intervinieron en su ordenador 6.780 álbumes musicales, 204 películas y 172 juegos de ordenador. La Audiencia Provincial absolvió al acusado de un delito contra la propiedad intelectual.

${ }^{16}$ La última resolución que se conoce en la fecha de escribir este artículo es el Auto de 26 de abril de 2012 de la Audiencia Provincial de Barcelona por el que se archivó la denuncia de ADESE contra un establecimiento que vendía programas de software utilizados para superar las barreras o códigos de protección que poseen los videojuegos y consolas originales.

${ }^{17}$ El Juzgado de lo Mercantil n ${ }^{\circ} 5$ de Madrid desestimó la demanda de la SGAE contra la asociación cultural Ladinamo mediante sentencia de fecha 2 de febrero de 2006. Uno de los argumentos del letrado de la entidad de gestión fue el de que estaba prohibido bajar música de internet. El caso Ladinamo fue el primero de numerosos casos más. Puede verse una relación de resoluciones judiciales en <http://derecho-internet.org/svn/procedimientos-libres/copyleft $>$. Fecha de consulta: 29 de diciembre de 2012.

${ }^{18}$ Se trata de las universidades Autónoma de Barcelona y Carlos III de Madrid. Los procedimientos actualmente se hallan subjudice ante los Juzgados de lo Mercantil números 2 de Barcelona y 11 de Madrid, respectivamente. 
de Justicia de la Unión Europea ${ }^{19}$. Además, la industria y las entidades de gestión han litigado contra las webs de enlaces a archivos en redes $\mathrm{p} 2 \mathrm{p}^{20}$, contra los desarrolladores de redes $\mathrm{p} 2 \mathrm{p}^{21}$, contra la caché del buscador Google ${ }^{22}$ y contra la compañía Telefónica solicitando los datos personales de quienes se conectaban a las redes $\mathrm{p} 2 \mathrm{p}^{23}$.

- (7): Ruido. En este supuesto los titulares de los derechos realizan una actividad consistente en invadir las redes con archivos basura que tienen el mismo nombre y peso del archivo original para desincentivar que los usuarios utilicen las redes $\mathrm{p} 2 \mathrm{p}$.

Características comunes a los anteriores litigios son la asimetría en los intervinientes en los procedimientos, puesto que se trata de importantes compañías transnacionales que pleitean contra particulares, así como la asimetría de las reglas procesales: en los litigios en ámbito civil sobre propiedad intelectual se invierte la carga de la prueba, concediéndose a las entidades de gestión por parte de la legislación y de la jurisprudencia una presunción de que representan a todos los

${ }^{19}$ Sentencia de 21 de octubre de 2010 de la Sala Tercera del Tribunal de Justicia de la Unión Europea. A pesar de ser declarado ilegal el pago por las Administraciones públicas, empresas y profesionales del coloquialmente llamado canon digital, el Real Decreto 1657/2012, de 7 de diciembre, por el que se regula el procedimiento de pago de la compensación equitativa por copia privada con cargo a los Presupuestos Generales del Estado, Boletín Oficial del Estado número 295 de 8 de diciembre de 2012, establece sorprendentemente en su Disposición transitoria primera que serán irrecuperables las cantidades pagadas por los equipos, aparatos y soportes materiales de reproducción adquiridos con anterioridad al 1 de enero de 2012. En definitiva, se dispone en una norma nacional de rango reglamentario lo contrario de lo ordenado por el Tribunal de Justicia de la Unión Europea.

${ }^{20}$ El auto de fecha 11 de septiembre de 2008 de la Audiencia Provincial de Madrid archivó definitivamente la acusación penal seguida contra los administradores de la web Sharemula por parte de SGAE, Microsoft, Promusicae, EGEDA, Columbia Tristar Home Entertainment y Cía, SRC, The Walt Disney Company Iberia, Twentieth Century Fox Home Entertainment España S.A., Warner Home Video, Lauren Films Video Hogar S.A., Manga Films S.L., Universal Pictures (Spain) S.L., Paramount Home Entertainment (Spain) S.L., Twentieth Century Fox Film Corporation, Walt Disney Enterprises Inc., Columbia Pictures Industries Inc., Tristar Pictures, Sony Pictures Classic Inc., Mandalay Entertainment, Metro Goldwin Mayer Studios Inc., Orion Pictures Corporation, Paramount Pictures Corporation, Universal City Studios y Time Warner Entertainment Company L. New Line Productions Inc.

${ }^{21}$ La sentencia de 25 de noviembre de 2011 del Juzgado de lo Mercantil n 4 de Madrid desestimó la demanda interpuesta por Sony BMG Music Entertainment Spain S.A., Warner Music Spain S.A., Universal Music Spain S.L., EMI Music Spain S.A. y Promusicae contra un desarrollador de software de redes $\mathrm{p} 2 \mathrm{p}$.

${ }^{22}$ La sentencia de 17 de septiembre de 2008 de la Audiencia Provincial de Barcelona desestimó la demanda interpuesta por el administrador de una web contra Google por la copia caché que el buscador conserva.

${ }^{23}$ La sentencia de 29 de enero de 2008 de la Gran Sala del Tribunal de Justicia de la Unión Europea desestimó las pretensiones de la patronal de la industria fonográfica, Promusicae. 
titulares de los derechos ${ }^{24}$, sin más requisito que aportar al inicio del proceso copia de sus estatutos y certificación acreditativa de su autorización administrativa otorgada en este caso por el Ministerio de Cultura ${ }^{25}$. La inversión de la carga de la prueba implica, en palabras de una resolución judicial ${ }^{26}$, que hemos caído jurisprudencialmente en un monopolio probatorio [en] favor de la $S G A E^{27}$.

Desde la perspectiva de la innovación, no cabe duda de que este sistema es contrario a cualquier desarrollo tecnológico que se realice fuera del consentimiento de las corporaciones ya que el problema práctico no es que un juzgado otorgue a un innovador la razón, sino el tiempo que tardará en hacerlo, los recursos que el innovador deberá destinar a su defensa legal desviándolos de otros fines más productivos y el padecimiento humano que produce el matonismo procesal practicado por las corporaciones y los organismos de su entorno, donde incluimos alguna entidad de gestión $^{28}$, que continúan pleiteando aunque hayan perdido casos análogos anteriores.

\section{Las soluciones en pro del conocimiento libre}

Las soluciones que se han propuesto para evitar la limitación a la libre transmisibilidad del conocimiento han tomado dos guías: la del código legal y la del código informático.

En lo que se refiere al código legal, sobresale la iniciativa de la estandarización de las licencias libres, de las que las más conocidas son, para código informático ${ }^{29}$, las licencias GPL (Licencia General Pública) y BSD (Berkeley Software Distribution) y, para obras literarias, artísticas o científicas, las licencias Creative Commons. La piedra angular de las licencias de propiedad intelectual libre consiste en que, en ausencia de obligación normativa, el autor de una obra tiene el

\footnotetext{
${ }^{24}$ No es éste el único tratamiento procesal favorable. Gozan de medidas cautelares desarrolladas ad hoc para sus intereses, régimen de control de aduanas y juzgados especializados (en España, los Juzgados de lo Mercantil).

${ }^{25}$ Cfr. artículo 150 TRLPI. Vid. Montero (1997, 73-74) y Marín (2007).

${ }^{26}$ Se trata de la sentencia ${ }^{\circ} 166 / 2008$ de 29 de diciembre de 2008 del Juzgado de lo Mercantil $\mathrm{n}^{\circ} 7$ de Madrid.

${ }^{27} \mathrm{Si}$ bien la SGAE es la entidad de gestión de derechos de propiedad intelectual más conocida, existen otras siete entidades más: CEDRO, AISGE, AIE, DAMA, AGEDI, EGEDA y VEGAP.

${ }^{28}$ No olvidemos que miembros de la directiva anterior de la SGAE se hallan actualmente imputados por apropiación indebida de los derechos de los socios en el procedimiento ante el Juzgado Central de Instrucción $n^{\circ} 5$ de la Audiencia Nacional, Diligencias Previas 90/2010. El Ministerio de Cultura nunca ha ejercitado su potestad de control de las entidades de gestión, por mucho que se le haya solicitado por escrito por parte de algún autor.

${ }^{29}$ Para un estudio de los diferentes tipos de licencias libres para código informático vid. Lindberg (2008).
} 
derecho exclusivo de decidir quién puede copiar, distribuir, difundir o transformar la misma ${ }^{30}$, por lo que si expresa públicamente mediante una licencia libre estándar que otorga alguno de esos derechos a terceros, se entienden concedidos los permisos y, por tanto, cualquiera puede hacer con la obra los usos que el autor ha anunciado públicamente. De esta manera se vence el sistema de permisos y la presunción de que si un autor nada ha dicho de su obra, se entienden todos los derechos reservados. El conocimiento libre, al adjuntar la licencia libre a la obra, proclama de antemano qué permisos están ya concedidos, dándose así la vuelta a los fines de la normativa de la propiedad intelectual, que se fundamenta en el control de la copia para rentabilizarla económicamente, y propugnando la difusión de la obra como aportación a una riqueza común.

La legislación es deficitaria en implementaciones en pro del conocimiento libre por lo que las soluciones se han tomado desde la práctica descrita en el párrafo anterior. Si bien una de las reivindicaciones de los partidarios del conocimiento libre es que el conocimiento producido con dinero público ha de ser de libre acceso, no parece éste ser el camino que toma la legislación, siendo pertinente la pregunta de Lafuente y Alonso (2011, 198): ¿Cómo hemos organizado una empresa tan ruinosa y abusiva para los intereses públicos? En nuestro país, dos normas ${ }^{31}$ transfieren la propiedad intelectual de las obras de los investigadores a los centros de los que dependen si bien no señalan si el centro ha de $l i-$ berar esa propiedad, por mucho que nuestra Ley de la Ciencia, la Tecnología y la Innovación contenga en su artículo 37 la regulación de la difusión del acceso abierto, de redacción altamente confusa y técnicamente defectuosa ${ }^{32}$.

Pero no toda información se halla sometida a regulación de propiedad intelectual (en el sentido amplio anglosajón comprensivo también de nuestra propie-

${ }^{30}$ Cfr. artículo 17 del TRLPI. Vid. Rivero (2007).

${ }^{31}$ Se trata del artículo 80 apartado 5 de la Ley Orgánica 6/2001, de 21 de diciembre, de Universidades, Boletín Oficial del Estado, número 307 de 24 de diciembre de 2001, y del artículo 54.2 de la Ley 2/2011, de 4 de marzo, de Economía Sostenible, Boletín Oficial del Estado, número 55 de 5 de marzo de 2011.

${ }^{32}$ El apartado 6 del artículo 37 de la Ley de la Ciencia, la Tecnología y la Innovación (Ley 14/2011, de 1 de junio, Boletín Oficial del Estado, número 131 de 2 de junio de 2011) establece la excepción de que no será obligatorio el acceso abierto «cuando los derechos sobre los resultados de la actividad de investigación, desarrollo e innovación sean susceptibles de protección». Olvida, o confunde, que todas las obras literarias, artísticas o científicas, incluso aquellas en las que el autor permite su libre copia, distribución, difusión y transformación, son susceptibles de protección. La confusión terminológica proviene de una neolengua de algunas de las entidades de gestión interesadas en la confusión entre los conceptos de obras protegidas y obras gestionadas: todas las obras están protegidas, pero no todas están gestionadas por las entidades de gestión de la propiedad intelectual. Confundir ambos conceptos les dotaría de potestad universal sobre todas las obras de propiedad intelectual y no sólo sobre las de sus socios. 
dad industrial), sino que encontramos elementos informacionales que por no tratarse de obras creativas quedan excluidas de esta regulación. Los ejemplos paradigmáticos son los datos meteorológicos o cartográficos, regulados por la normativa de la reutilización de la información del sector público. La solución que se ha dado en este caso por parte de los partidarios del conocimiento libre ha sido la de generar una definición de qué se entiende por abierto. Se han tenido que vencer problemas terminológicos entre los términos abierto y libre -Lindberg $(2008,154)$ y Vidal $(2010)$ - que nacen debido a la polisemia en inglés del término free software, traducible tanto por software libre como por software gratuito. Para evitar que pudiera pensarse que se trataba de un software gratuito, comenzó a utilizarse el término open source, del que posteriormente ha derivado la utilización de open como sinónimo de libre. Para homogeneizar conceptos por encima de las regulaciones, la Open Knowledge Foundation británica impulsó la iniciativa Open Definition $^{33}$, en la que señala ${ }^{34}$ que una obra es abierta [libre] si su forma de distribución satisface una serie de condiciones que podemos sintetizar en que la obra sea libremente reutilizable con independencia del estatuto jurídico al que se halle sometida. De esta manera, las denominaciones open data, open science, open content y open access deberemos entenderlas en su acepción de libres.

Código jurídico y código informático trabajan tanto conjunta como opuestamente. Si bien el código jurídico establece las condiciones que regulan el código informático, éste se utiliza tanto para instituir la arquitectura tecnológica necesaria para facilitar el almacenamiento y la transmisión de las obras (el universo de gestores de contenidos, wikis, repositorios documentales...) como para hacer de imposible aplicación las normas de propiedad intelectual que regulan las infracciones. De esta manera, una obra tecnológica protegida por la propiedad intelectual paradójicamente se utiliza como troyano ${ }^{35}$ de la propia parte del ordenamiento jurídico que la regula, hiriendo de muerte la aplicabilidad de la norma cuando ésta prohíbe la reutilización, como si fuera una venganza de una colectividad ${ }^{36}$ que trabaja reforzando la economía del don ${ }^{37}$ contra quienes utilizan los recursos públicos en favor de intereses privados tal y como vimos en el apartado supra sobre las dificultades para el desarrollo del conocimiento

${ }^{33}$ Vid. $<$ http://opendefinition.org/>. Fecha de consulta: 29 de diciembre de 2012.

${ }^{34}$ Para todos los requisitos, vid. <http://opendefinition.org/okd/espanol/>. Fecha de consulta: 29 de diciembre de 2012.

${ }^{35}$ Un troyano es un software malicioso que se ejecuta en un ordenador y que crea una puerta trasera que permite la administración remota del mismo.

${ }^{36}$ Acerca de las colectividades que realizan aportaciones de trabajo desinteresado en red, vid. Benkler (2006).

${ }^{37}$ Sobre la economía del don en el entorno digital, vid. Ortega y Rodríguez $(2011,39-43)$ y con carácter general, vid. Mauss (2007). 
libre. Podemos utilizar nuevamente el modelo de comunicación de Shannon para enumerar las obras jurídicas e informáticas que los hackers ${ }^{38}$ han escrito para facilitar la transmisión: software libre para que los emisores y receptores gestionen su sistema de emisión y recepción de información; en lo que respecta al canal de comunicación, se han diseñado redes $\mathrm{p} 2 \mathrm{p}$, instalado servidores que borran sus registros, diseñado e implantado redes privadas virtuales $\left(O p e n v p n^{39}\right)$, sistemas para nombres de dominio libres $\left(\right.$ Opendns ${ }^{40}$ ), redes wifi ciudadanas $\left(\right.$ Guifi.net $\left.^{41}\right)$; en lo referente a los mensajes, contenidos libres y, por último, con respecto a la codificación, protocolos (TCP/IP, UDP, email, FTP, SSH...), sistemas de cifrado $\left(\mathrm{GPG}^{42}\right)$ y sistemas de elusión de zonas comerciales y protecciones tecnológicas $\left(\mathrm{DeCSS}^{43}\right)$. De esta manera, los hackers se postulan como legisladores negati$v o s^{44}$ dotando a los usuarios de herramientas tecnológicas que éstos utilizan para incumplir las normas jurídicas que restringen la reutilización, haciendo caso omiso de los permisos de los titulares ${ }^{45}$.

\section{Cuestiones morales y politicas}

Una relevante cuestión moral acerca del conocimiento libre fue planteada por el profesor Eben Moglen en la conferencia que impartió el 29 de junio de $2003^{46}(2006,197)$ :

${ }^{38}$ Cualquier persona, sin necesidad de conocimientos informáticos, puede ser un hacker. Se utiliza el término hacker en su acepción originaria de experto, especialista, y sin la connotación de delincuencia a la que los medios de comunicación nos tienen acostumbrados. Sobre el término en su acepción originaria ver Sterling (1992, 50-51), Hafner y Markoff $(1995,11)$ y, especialmente, Levy (2001, 23).

${ }^{39} \mathrm{Vid} .<$ http://openvpn.net/>. Fecha de consulta: 29 de diciembre de 2012.

${ }^{40} \mathrm{Vid}$. < https://www.opendns.com/>. Fecha de consulta: 29 de diciembre de 2012.

${ }^{41}$ Vid. $<$ https://guifi.net/>. Fecha de consulta: 29 de diciembre de 2012.

${ }^{42}$ Vid. $<$ http://www.gnupg.org/>. Fecha de consulta: 29 de diciembre de 2012.

${ }^{43}$ Vid. $<$ http://www.lemuria.org/DeCSS/decss.html $>$. Fecha de consulta: 29 de diciembre de 2012.

${ }^{44}$ El concepto de legislador negativo proviene de la tradición constitucional y consiste en la facultad que tiene un tribunal de borrar del ordenamiento jurídico una norma que se considera no válida por ser contraria a una norma de rango superior. Sobre este concepto, vid. García de Enterría (1982, 121-137), Loewenstein (1983, 308-321), Kelsen (1983, 280).

${ }^{45}$ Sobre cómo la arquitectura del código informático modela las normas jurídicas, vid. Les$\operatorname{sig}(1999$ y 2009).

${ }^{46}$ Impartida en el seno de la IV Conferencia Anual sobre Ley y Tecnología, Universidad de Portland, Maine (EE.UU.). El texto original se halla en la página web personal del profesor Moglen: $<$ http://moglen.law.columbia.edu/publications/maine-speech.html $>$. Fecha de consulta: 29 de diciembre de 2012. 
Si puedo proporcionar todos los bienes de valor intelectual o estético a todo el mundo por el mismo precio que puedo proporcionar a cualquiera la primera copia de estos trabajos, ¿por qué es moral excluir a cualquiera de cualquier cosa? Si se puede alimentar a toda la población del planeta tierra con el coste de hornear una barra de pan y apretar un botón, ¿qué fuerza moral tendría cobrar más por el pan de lo que algunos podrían permitirse pagar por él?

El planteamiento del profesor Moglen no es sólo teórico sino que tiene aplicaciones prácticas importantes y actuales. Por ejemplo, el estado del arte de la tecnología permite que los libros de texto de los alumnos de las educaciones primarias y secundarias puedan ser ediciones digitales descargables de un repositorio en internet. Sin embargo no existe iniciativa política alguna para desarrollar esta práctica donde lo que ahorrarían los padres dejaría de ingresarlo la industria editorial ${ }^{47}$. De hecho, incluso, se cambian los textos de un año para otro para que los libros de un curso escolar no sean reutilizables en el siguiente. Parece que sería un caso paradigmático de intervención política máxime en tiempos de crisis y, sin embargo, nada se hace desde ninguna de las administraciones públicas para racionalizar este gasto familiar ${ }^{48}$.

Pero las implicaciones son más profundas puesto que imponer barreras económicas de entrada al conocimiento produce la exclusión social de individuos dentro de una sociedad o de grupos sociales o naciones enteras si la escala en la que nos movemos es la de la comunidad internacional (Smiers, 2006, 305312). Lo que está en la base de esta discusión es el derecho a la igualdad, al que podemos acercarnos desde dos perspectivas: la primera postura es la de una propiedad intelectual punitiva, ilimitada y perpetua, mediante el desarrollo de los derechos privados para cuya defensa se utilizan recursos públicos y defendiendo que sólo con este modelo es posible la inversión económica, la innovación, el progreso y las obras de calidad $^{49}$, mientras que la segunda

\footnotetext{
${ }^{47}$ El libro de texto de Lengua de $4^{\circ}$ de Primaria (niños de 8 y 9 años) de Editorial Anaya contiene el siguiente texto: «Busca el significado de pirata y piratear en el diccionario. Debate con tus compañeros acerca de las acciones de los piratas en la actualidad: secuestros de barcos o de aviones, robos de obras de arte (música, cine...), de datos personales por internet... ¿Qué piensas de aquellos que 'piratean' la música o el cine? ¿Te gustaría que te 'piratearan' un trabajo tuyo?» La referencia completa de esta obra es la siguiente: Carmen Bello Crespo, Tomás Bernal Regalado, Carlos Lluva Mera, José Luis Magarzo Jiménez, Pilar Zaragoza García (2012). En Linea, lengua, 4 Educación Primaria. Madrid, Editorial Anaya, ISBN 13: 978-84-678-1689-1.

${ }^{48}$ Es interesante señalar que las modificaciones introducidas en la normativa de propiedad intelectual se han realizado siempre mediante un consenso entre los partidos PP, PSOE y CiU, situación excepcional puesto que prácticamente no se ha obtenido consenso en ninguna otra parcela política.

${ }^{49}$ Nótese que los argumentos inversión y obras de calidad son permanentes en la terminología de los representantes de nuestro Ministerio de Cultura. Cfr. $<\mathrm{http} / /$ www.mcu.es/industrias/Ayudas/Ayudas inversion.html>. Fecha de consulta: 29 de diciembre de 2012.
} 
perspectiva es la del conocimiento libre, facilitando la reutilización, máxime si el conocimiento se ha generado con dinero público, y vertiendo las obras desinteresadamente en el procomún digital (Lafuente, 2007). Con su pregunta, el profesor Moglen está incidiendo en el derecho de igualdad pero precisamente en un campo clave para un sistema democrático, el de la educación. Nada ha cambiado en este sentido desde la paideia: El nexo entre democracia y educación está inscrito en el código genético de la democracia (Greppi, 2012, 149). El problema no escapa a Nussbaum (2010, 20-21), para quien [e]stamos en medio de una crisis de proporciones gigantescas y de enorme gravedad a nivel mundial [...] me refiero a una crisis que pasa prácticamente inadvertida [...] la crisis mundial en materia de educación. Sólo mediante un sistema que haga la cultura y educación más accesible podemos satisfacer el ideal de permitir la igualdad de oportunidades a todas las personas con independencia del entorno social, económico o de cualquier otra naturaleza del que procedan, esto es, sólo así estamos en condiciones de favorecer la circulación de las élites ${ }^{50}$, promoviendo el gobierno por los mejores y evitando la perpetuación de estirpes en el poder sin más mérito que el de la adscripción a una familia.

Si la educación es esencial para la democracia, entonces el conocimiento libre podrá ayudarnos a reflexionar sobre los dos problemas que Bermudo (2001, 63), citando a Tenzer, asigna a la filosofía política: la fijación de los fines o ideales, esto es, los valores, y la fijación del procedimiento u ordenación interna del debate colectivo. Bermudo añade una tercera cuestión, la fijación del orden democrático, habida cuenta de que las dos primeras son políticamente neutras y podrían cumplirse respecto a cualquier orden político $(2001,64)$. Las tres cuestiones, como veremos a continuación, tienen su reflejo en normas jurídicas y se interpretan mediante resoluciones judiciales ${ }^{51}$, que se hallan exentas de propiedad intelectual ${ }^{52}$, siendo inconcebible un sistema político democrático en el que no se pudieran copiar o difundir libremente. Sólo si la ley puede copiarse pueden obtenerse dos de las premisas básicas de un sistema democrático: la seguridad jurídica y la publicidad de las

\footnotetext{
${ }^{50}$ Para una introducción a la teoría de las élites, vid. Morán (1994).

${ }^{51}$ En España tuvimos el «Acuerdo de 28 de octubre de 2010, del Pleno del Consejo General del Poder Judicial (CGPJ), por el que se aprueba el Reglamento 3/2010, sobre reutilización de sentencias y otras resoluciones judiciales», Boletín Oficial del Estado número 282 de 22 de noviembre de 2010. Este reglamento fue declarado nulo por una sentencia de 28 de octubre de 2011 del Pleno de la Sala Tercera del Tribunal Supremo que declaró que el CGPJ no tiene competencias para dictar un reglamento sobre la reutilización de las resoluciones judiciales.

${ }^{52}$ Cfr. artículo 13 TRLPI. Vid. Bercovitz (2007b), si bien el análisis que realiza es meramente a los efectos de la propiedad intelectual y no del ordenamiento jurídico como núcleo estructural de la sociedad.
} 
normas, que tienen como misión la interdicción de la arbitrariedad de los poderes públicos. (De la Cueva, 2006, 142) ${ }^{53}$. Por tanto, parafraseando a Greppi, también el conocimiento libre está inscrito en el código genético de la democracia. Ahora bien, donde nos desenvolvemos en el actual momento histórico es en el tercer entorno ${ }^{54}$ (Echeverría 1999, 48-57 y 2012, 184-187), por lo que es dentro de este contexto tecnocientífico donde deberemos analizar las tres cuestiones.

Comenzaremos por la fijación del orden democrático. Existe un consenso en que nos hallamos en una era de déficit democrático de prevalencia de intereses privados frente a los públicos sintetizado, como ya expresamos, en una perversa relación entre el dinero, la información y la política: dinero para hacer política e información, información para hacer dinero y política, política para hacer dinero e información (Ferrajoli, 2010a, 271, y 2011, 53), donde la doxa se ha elevado a categoría de absoluto, donde la verdad tiene una dificultad real en hacerse visible y donde el ideal de un interés común situado por encima y más allá de los intereses de grupo [...] es una ilusión metafisica o, mejor, metapolítica (Kelsen, 2006, 80). La agenda viene señalada desde los medios de comunicación social, actores en un mercado altamente concentrado en manos de unos $\operatorname{pocos}^{55}$. Mal puede fijarse un orden democrático de esta manera y mucho menos realizar innovación en pro del bien común cuando la información desde la que se parte es engañosa y en un espacio público en el que se negocia el mundo común, pero con dos graves problemas: tanto con las conducciones, casi exclusivamente reducidas al sistema de los medios de comunicación, como con la fuente de energía, los ciudadanos, poco atentos en general a lo que debería concernirles (Vallespín, 2012, 140) lo que, obviamente, generará déficits en la formación de la opinión pública ${ }^{56}$. Para evitarlos, frecuentemente se trata el tema del recurso a los expertos para la formación de

${ }^{53}$ El sector jurídico es una gran demostración empírica de cómo parte relevante de una población puede vivir de un conocimiento simbólico no sometido a propiedad intelectual y en continua ebullición creativa (jueces, parlamentarios, políticos, registradores, notarios, abogados, procuradores, funcionarios, policías, delincuentes...).

${ }^{54}$ El primero de los entornos señalado por Echeverría es el medio ambiente natural (1999, 27); el segundo entorno ya no es natural, sino cultural y social, y puede ser denominado entorno urbano (1999, 41); el tercer entorno es uno de los resultados de la tecnociencia $(1999,49)$, es un espacio básicamente artificial y está posibilitado por una serie de tecnologías, entre las cuales mencionaremos siete: el teléfono, la radio, la televisión, el dinero electrónico, las redes telemáticas, los multimedia y el hipertexto $(1999,48)$.

${ }_{55}$ Vid. Castells (2009, 112-130).

${ }^{56}$ Sobre la formación de opinión, vid. Sartori (2010, 169-200). Sobre la opinión pública, vid. Habermas (2006). 
una correcta opinión, lo que se ha estudiado desde las dos parcelas de la ciencia política y de los estudios de Ciencia, Tecnología y Sociedad (CTS $)^{57}$, donde se plantean los problemas de la legitimidad y de la extensión: legitimidad en cuanto a si todos tenemos derecho a la participación en la toma de decisiones ${ }^{58}$ y extensión en cuanto a hasta qué punto debe extenderse dicha participación (Aibar, 2012, 310). En definitiva, se trata nuevamente del derecho a la igualdad, la democracia moderna se caracteriza por la universalización del derecho de participación política (Bovero, 2002, 26), y del viejo problema prometeico en Platón (2009, 322a-323d) pero con tecnologías nuevas y aunque éstas no sean salvíficas (Winner, 2008), sí que permiten un mejor acceso al conocimiento permitiendo que las políticas públicas sobre cualquier innovación puedan ser más reflexivas y transparentes.

Sobre la fijación de los fines o ideales, los valores, no nos cabe duda que los derechos fundamentales constituyen el único dogma permisible. Ahora bien, en una sociedad que se desarrolla en el tercer entorno, tomando como inspiración a Quintanilla $(2005,277)$ y como guía a Ferrajoli, encontraremos derechos fundamentales de cultura tecnológica incorporada y de cultura tecnológica no incorporada, esto es, derechos de cultura tecnológica informacional incorporada o no incorporada, lo que traducimos en derechos cuyo núcleo es o no informacional. La libertad de expresión, el derecho a la información o el derecho de petición son puramente informacionales, pero el derecho de huelga, sin perjuicio de que pueda utilizarse información entre los ejercientes del mismo, no tiene un núcleo informacional ya que su contenido sustancial consiste en dejar de trabajar. Esta división nos permite, y nos exige, plantearnos una relectura de los derechos fundamentales de carácter informacional en el contexto del tránsito de la ciudadanía urbana e industrial [...] a la infociudadanía (Echeverría, 2003, 90). Los derechos fundamentales de carácter no informacional no necesitarían una relectura tan exhaustiva puesto que su tradición no se ve afectada por un cambio de entorno, mientras que los informacionales requerirán una redefinición ${ }^{59}$. Ferrajoli (2010b, 40, y 2011, 35-36) nos ofrece una tipología de cuatro grupos de derechos fundamentales

${ }^{57}$ Para un compendio sobre los estudios CTS, vid. Aibar y Quintanilla (ed.) (2012).

${ }^{58}$ Existen, como no podía ser de otra manera, voces a favor y en contra. Señala Schawrtz Cowan $(1997,223)$ que la opinión de los expertos no es desinteresada, y dado que todos los homo faber no podemos escapar de las consecuencias de la tecnociencia, todos tenemos derecho a estar informados. En el mismo sentido, Lafuente y Alonso (2011, 125-141).

${ }^{59}$ Este parece ser el camino que se está tomando. En este sentido, véase Organización de Naciones Unidas (2011, 22): Given that the Internet has become an indispensable tool for realizing a range of human rights, combating inequality, and accelerating development and human progress, ensuring universal access to the Internet should be a priority for all States. 
que obtiene mediante la combinación de las categorías de derechos de la personalidad y derechos de la ciudadanía, por un lado, y de derechos primarios o secundarios, por otro: (i) los derechos humanos -vida, integridad, libertad personal, libertad de conciencia y de expresión, salud, educación, garantías penales y procesales-(ii) los derechos públicos -residencia, circulación, asociación, reunión, trabajo, derecho a la subsistencia y asistencia a discapacitados- (iii) los derechos civiles - potestad negocial, libertad contractual, libertad de elección de trabajo, libertad de empresa, derecho a accionar en juicio y en general los derechos relacionados con el mercado-y (iv) los derechos políticos -derechos de sufragio activo y pasivo, derecho al acceso a los cargos públicos y en general los relativos a la autonomía, representación y democracia políticas-. Estos son los derechos cuyo ejercicio tendremos que verificar en el tercer entorno y, en caso de ser de información incorporada, reflexionar sobre ellos.

Y, finalmente, acerca de la ordenación interna del debate colectivo, es difícil trazar una separación nítida con las dos cuestiones anteriores. Su ligazón con los valores es íntima: los derechos de información, participación, acceso a cargos públicos, petición, asociación y reunión presumen o suponen un sistema organizativo. Y su nexo con la fijación del orden democrático, lo encontramos a través la permanente búsqueda de que el debate colectivo debe realizarse en condiciones de transparencia pública, ejercitando el derecho de participación (de naturaleza informacional) que se manifiesta mediante el derecho a saber y busca la interdicción de la corrupción y el desarrollo de una sociedad abierta democrática. Señala Nadal (2012) que la sociedad abierta popperiana ${ }^{60}$ no garantiza la existencia de un régimen democrático, sino únicamente la posibilidad de falsabilidad de los actos de los gobernantes, de tal manera que los requisitos señalados por este autor si bien son importantes, lo son como algo necesario pero no suficiente: la sociedad abierta de Popper, continúa esta autora, incumple el principio de isegoría ${ }^{61}$, esencial para considerar que una sociedad es participativa en el sentido pleno. No es lo mismo participar en la destitución de malos gobernantes que participar en los asuntos públicos entendido como toma de decisiones. La participación exige retorno de la información y como tal derecho político fundamental no debe estar sometido a un envoltorio de propiedad intelectual restrictiva ${ }^{62}$. Pero este derecho a la participación ha de realizarse buscando un poder en público. Utilizo

\footnotetext{
${ }^{60}$ Vid. Popper (2010).

${ }^{61}$ Sobre la redefinición del concepto de isegoría acorde con las TIC, vid. Nadal y De la Cueva (2012).

${ }^{62}$ Sobre la problemática de que derechos ordinarios como la propiedad intelectual enmarquen derechos fundamentales, vid. De la Cueva (2012).
} 
esta expresión sintética para indicar todos aquellos mecanismos institucionales que obligan a los gobernantes a tomar sus decisiones a la luz del día, y permiten a los gobernados «ver» cómo y dónde se toman dichas decisiones (Bobbio, 2009, 418). La transparencia ${ }^{63}$, señalan Solimano, Tanzi y Del Solar $(2008,97)$, es parte central de reformas especificas tendentes a modernizar el Estado y controlar el oportunismo y la discrecionalidad. Y así es, puesto que no debemos sólo pensar que la transparencia es un elemento relacional entre gobernantes y gobernados sino, también entre administraciones públicas, puesto que el conocimiento libre en su faceta de información del Estado, al hacer más fluida la información, permite una mejor gestión de la res publica. Hacer más observables los procesos, continúan estos autores, favorece una mayor probidad y genera un más alto respeto del interés público sobre intereses particulares.

Para concluir, hemos de señalar que existen muchos motivos para reflexionar. Nos hallamos ante una nueva transformación del Estado en la que los aspectos morales y políticos del conocimiento, como hemos podido ver, son demasiado importantes para que los dejemos en manos de quienes, no nos cansaremos de decirlo, utilizan recursos públicos para sus fines privados y generan un conocimiento espurio. Hablar de conocimiento libre no es, por tanto, hablar de una cuestión de propiedad intelectual sino de un elemento estructural de la democracia: la información como soporte para el conocimiento, una parcela donde el silencio resulta ser un silencio cómplice ${ }^{64}$.

\section{REFERENCIAS BIBLIOGRÁFICAS}

AEVAL - Agencia Estatal de Evaluación de las Políticas Públicas y la Calidad de los Servicios (2008). Evaluación del Sistema de Gestión Colectiva de los Derechos de Propiedad Intelectual. Madrid. Documento accesible en línea: $<$ http://www.aeval.es/ comun/pdf/evaluaciones/E12B.pdf $>$. Fecha de consulta: 29 de diciembre de 2012.

Aibar, E. (2012). «El público en las decisiones científico-tecnológicas», en Aibar, E., Quintanilla, M.A. (ed.). Ciencia, Tecnología y Sociedad, pp. 303-323. Madrid: Editorial Trotta/CSIC

${ }^{63}$ Sobre las relaciones entre corrupción y transparencia, vid. Solimano, A., Tanzi, V, Del Solar (2008, 94-104).

${ }^{64} \mathrm{Y}$, como nota final, por si cupiera duda, hemos de señalar que no estamos en contra de la propiedad intelectual, sino en contra del injusto apropiacionismo constante y sistemático de los derechos de los más débiles realizado por los más fuertes. 
Aibar, E. y Quintanilla, M.A. (ed.). (2012). Ciencia, Tecnología y Sociedad. Madrid: Editorial Trotta/CSIC.

Aleksander, I. (2004). «Comprender la información bit a bit. Las ecuaciones de Shannon». En Farmelo, G. (ed.). Fórmulas elegantes, pp. 189-212. Barcelona: Tusquets.

Benkler, Y. (2006). The Wealth of Networks. How Social Production Transforms Markets and Freedom. New Haven y Londres: Yale University Press. Documento accesible en línea: < http://www.benkler.org/Benkler_Wealth_Of_Networks.pdf>. Fecha de consulta: 29 de diciembre de 2012.

Bercovitz, R. (2007a). Comentario al artículo 10.1 del TRLPI, en Bercovitz, R. (coordinador), Comentarios a la Ley de Propiedad Intelectual. $3^{\mathrm{a}}$ ed., pp. 151-164. Madrid: Tecnos.

Bercovitz, R. (2007b). Comentario al artículo 13 del TRLPI, en Bercovitz, R. (coordinador), Comentarios a la Ley de Propiedad Intelectual. $3^{\mathrm{a}}$ ed., pp. 151-164. Madrid: Tecnos.

Bermudo, J. M. (2001). Filosofía política. I. Luces y sombras de la ciudad. Barcelona: Ediciones del Serbal.

Bessen, J. y Meurer, M.J. (2008). Patent Failure: How Judges, Bureaucrats, and Lawyers Put Innovators at Risk. Princeton (Nueva Jersey, EE.UU.): Princeton University Press.

Bessen, J. y Ford, J. L. (2011). «The Private and Social Costs of Patent Trolls». Boston University School of Law, Law and Economics Research Paper, No. 11-45. septiembre de 2011. Documento accesible en línea: <http://ssrn.com/abstract $=1930272>$. Fecha de consulta: 29 de diciembre de 2012.

Bobbio, N. (2009). Teoría general de la política. Madrid: Editorial Trotta.

Bovero. M. A. (2002). Una gramática de la democracia. Contra el gobierno de los peores. Madrid: Editorial Trotta.

Capella, J.R. (2008). Fruta prohibida. Una aproximación histórico-teorética al estudio del derecho y del estado. Madrid: Editorial Trotta.

Castells, M. (2009). Comunicación y poder. Madrid: Alianza Editorial.

CNC - Comisión Nacional de la Competencia (2009). Informe sobre la gestión colectiva de derechos de propiedad intelectual. Madrid. Documento accesible en línea: $<$ http://www.cncompetencia.es/Inicio/Informes/InformesyEstudiossectoriales/tabid/ 76/default.aspx?entryid=125519\&command=core_download\&method=attachment> Fecha de consulta: 29 de diciembre de 2012.

De la Cueva, J. (2006). «El Derecho es Copyleft. O la libertad de copiar las leyes», en Copyleft. Manual de uso. Madrid: Traficantes de Sueños, pp. 141-158. Documento accesible en línea: <http://www.traficantes.net/index.php/trafis/editorial/catalogo/otras/ copyleft_manual_de_uso__ $>$. Fecha de consulta: 29 de diciembre de 2012. 
De la Cueva, J. (2012). Copyright reform, commons, free knowledge and democracy. Conferencia en el debate del Grupo parlamentario S\&D 'Copyright: what is broken, how to mend it?' en el Parlamento Europeo, Bruselas, Bélgica. Documento accesible en línea: <http://derecho-internet.org/files/2012-10-18_european-parliament _ $0 . p d f>$. Fecha de consulta: 29 de diciembre de 2012.

Drahos, P. y Braithwaite, J. (2002). Information feudalism. Who Owns the Knowledge Economy? Nueva York: New Press.

Echeverría, J. (1999). Los señores del aire: Telépolis y el Tercer Entorno. Barcelona: Destino.

Echeverría, J. (2003). «Tecnociencias de la información y participación ciudadana». Isegoría, número 28, 2003, pp. 73-92. Documento accesible en línea: <http://isegoria.revistas.csic.es/index.php/isegoria/article/view/507/507>. Fecha de consulta: 29 de diciembre de 2012.

Echeverría, J. (2012). «¿Democracia en internet?», en Champeau, S. y Innerarity, D. (comps.). Internet y el futuro de la democracia, pp. 179-199. Barcelona: Paidós.

Ferrajoli, L. (2010a). Democracia y garantismo. $2^{\mathrm{a}}$ ed. Madrid: Editorial Trotta.

Ferrajoli, L. (2010b). Derechos y garantías. La ley del más débil. $7^{\mathrm{a}}$ ed. Madrid: Editorial Trotta.

Ferrajoli, L. (2011). Poderes salvajes. La crisis de la democracia constitucional. Madrid: Editorial Trotta.

García de Enterría, E. (1982). La constitución como norma y el Tribunal Constitucional. Madrid: Cívitas.

Greppi, A. (2012). La democracia y su contenido. Madrid: Editorial Trotta.

Gurrutxaga, A. y Echeverría, J. (2012). La luz de la luciérnaga. Diálogos de innovación social. Madrid: Plaza y Valdés.

Habermas, J. (2006). Historia y crítica de la opinión pública. La transformación estructural de la vida pública. Barcelona: Editorial Gustavo Gili.

Hafner, K. y Markoff, J. (1995). Ciberpunk. Outlaws and Hackers on the Computer Frontier. Nueva York (EE.UU.): Touchstone.

Hess, C. y Ostrom, E. (2007). «Introduction: An Overview of the Knowledge Commons», en Hess, C. y Ostrom, E. (ed.). Understanding Knowledge as a Commons. From Theory to Practice, pp. 3-26. Massachusetts (EE.UU.): The MIT Press.

Horkheimer, M. y Adorno, T. (2009). Dialéctica de la Ilustración. $9^{\mathrm{a}}$ ed. Madrid: Editorial Trotta.

Kelsen, H. (1983). Teoría pura del Derecho. México: Universidad Nacional Autónoma.

Kelsen, H. (2006). De la esencia y valor de la democracia. Oviedo: KRK. 
Lafuente, A. (2007). Los cuatro entornos del procomún. Archipiélago. Cuadernos de Crítica de la Cultura, noviembre 2007, número 77-78, pp. 15-22. Documento accesible en línea: <http://digital.csic.es/bitstream/10261/2746/1/cuatro_entornos_procomun.pdf> Fecha de consulta: 29 de diciembre de 2012.

Lafuente, A. y Alonso, A. (2011). Ciencia expandida, naturaleza común y saber profano. Bernal (Buenos Aires): Universidad Nacional de Quilmes.

Lessig, L. (1999). Code and other laws of cyberspace. Nueva York: Basic Books.

Lessig, L. (2005). Por una cultura libre. Cómo los grandes grupos de comunicación utilizan la tecnología y la ley para clausurar la cultura y la creatividad. Madrid: Traficantes de Sueños.

Lessig, L. (2009). El código 2.0. Madrid: Traficantes de Sueños.

Levy, S. (2001). Hackers. Heroes of the Computer Revolution. Nueva York (EE.UU.): Penguin Books.

Lindberg, V. (2008). Intellectual Property and Open Source. A Practical Guide to Protecting Code. Sebastopol (California, EE.UU.): O’Reilly.

Loewenstein, K. (1983). Teoría de la Constitución. Barcelona: Ariel.

Marín, J. J. (2007). Comentario al artículo 150 del TRLPI, en Bercovitz, R. (coordinador), Comentarios a la Ley de Propiedad Intelectual. $3^{\mathrm{a}}$ ed., pp. 1831-1857. Madrid: Tecnos.

Mauss, M. (2007). Essai sur le don. París: PUF.

McCurdy, D. P. (2009). «Patent Trolls Erode the Foundation of the U.S. Patent System», en Science Progress. Documento accesible en línea: <http://scienceprogress.org/2009/01/patent-trolls-erode-patent-system/>. Fecha de consulta: 29 de diciembre de 2012.

Moglen, E. (2006). «Liberar la mente: el software libre y el fin de la cultura privativa», en Copyleft. Manual de uso, pp. 193-215. Madrid: Traficantes de Sueños, Documento accesible en línea: <http://www.traficantes.net/index.php/trafis/editorial/ catalogo/otras/copyleft_manual_de_uso_1 $>$. Fecha de consulta: 29 de diciembre de 2012.

Montero, J. (1997). La legitimación colectiva de las entidades de gestión de la propiedad intelectual. Granada: Editorial Comares.

Morán, M. L. (1993). «La teoría de las élites», en Vallespín, F. (ed.). Historia de la Teoría Política, 5. Madrid: Alianza Editorial.

Nadal, H. (2012). «El retorno de la información en el derecho de participación y en las políticas de open government», en Clovis G. (organizador). Direitos Humanos e participacâo politica. Vol. III, pp. 261-304. Porto Alegre (Brasil): Imprensalivre.

Nadal, H. y De la Cueva, J. (2012). «Redefiniendo la isegoría: open data ciudadanos», en Cerrillo i Martínez, A., Peguera, M., Peña-López, I., Pifarré de Moner, M.J., \& 
Vilasau Solana, M. (coords.). Retos y oportunidades del entretenimiento en línea. Actas del VIII Congreso Internacional, Internet, Derecho y Política. Universitat Oberta de Catalunya, Barcelona 9-10 Julio, 2012, pp. 283-300. Barcelona: UOCHuygens Editorial. Documento accesible en línea: $<$ http://openaccess.uoc.edu/webapps/o2/bitstream/10609/15121/6/IDP_2012.pdf $>$. Fecha de consulta: 29 de diciembre de 2012.

Nussbaum, M. (2010). Sin fines de lucro. Por qué la democracia necesita de las humanidades. Madrid: Katz Editores.

Organización de Naciones Unidas. (2011). Asamblea General. Consejo de Derechos Humanos. Sesión número 17, de 16 de mayo de 2011. Report of the Special Rapporteur on the promotion and protection of the right to freedom of opinion and expression, Frank La Rue . Documento accesible en línea: <http://www2.ohchr.org/ english/bodies/hrcouncil/docs/17session/a.hrc.17.27_en.pdf $>$. Fecha de consulta: 29 de diciembre de 2012.

Ortega, F. y Rodríguez, J. (2011). El potlatch digital. Wikipedia y el triunfo del procomún y el conocimiento compartido. Madrid: Cátedra.

Patterson, L. (1968). Copyright in Historical Perspective. Nashville, Tennessee (EE.UU.): Vanderbilt University Press.

Platón. (2009). Protágoras. Gorgias. Carta Séptima. Madrid: Alianza.

Popper, K. (2010). La sociedad abierta y sus enemigos. Madrid: Paidós Básica.

Posner, R. (2007). El análisis económico del derecho. $2^{\mathrm{a}}$ ed. México: Fondo de Cultura Económica.

Quintanilla (2005). Tecnología: un enfoque filosófico y otros ensayos de filosofía de la tecnología. México: Fondo de Cultura Económica.

Rivero, F. (2007). Comentario al artículo 17 del TRLPI, en Bercovitz, R. (coordinador), en Comentarios a la Ley de Propiedad Intelectual. $3^{\mathrm{a}}$ ed., pp. 269-283. Madrid: Tecnos.

Rose, M. (1993). Authors and Owners. The Invention of Copyright. Cambridge, Massachusetts (EE.UU.): Harvard University Press.

Sartori, G. (2010). Elementos de teoría política. Madrid: Alianza Editorial.

Sell, S. K. (2003). Private Power, Public Law. The Globalization of Intellectual Property Rights. Cambridge (Reino Unido): Cambridge University Press.

Shannon, C. (1948). «A Mathematical Theory of Communication», en The Bell System Technical Journal. Vol. 27, julio, octubre de 1948, pp. 379-423, 623-656. Documento accesible en línea: <http://www.mast.queensu.ca/ math474/shannon1948.pdf>. Fecha de consulta: 29 de diciembre de 2012.

Schwartz Cowan, R. (1997). A Social History of American Technology. Nueva York: Oxford University Press. 
Smiers, J. (2006). Un mundo sin copyright. Barcelona: Gedisa.

Smiers, J. (2008). Imagine... No Copyright. Barcelona: Gedisa.

Solimano, A., Tanzi, V. y Del Solar, F. (2008). Las termitas del Estado. Ensayos sobre corrupción, transparencia y desarrollo. Chile: Fondo de Cultura Económica.

Sterling, B. (1992). The Hacker Crackdown. Law and Disorder on the Electronic Frontier. Nueva York (EE.UU.): Bantam Books.

Tucker, C. (2011). Patent Trolls and Technology Diffusion. Documento accesible en línea: <https://papers.ssrn.com/sol3/papers.cfm?abstract_id=1976593>. Fecha de consulta: 29 de diciembre de 2012 .

Vaikhari, R. (2008). «The Effect of Patent Trolls on Innovation: A Multi-Jurisdictional Analysis», en Indian Journal of Intellectual Property Law. Vol. 1, diciembre de 2008, pp. 33-48. Documento accesible en línea: <http://ssrn.com/abstract= 1320553>. Fecha de consulta: 29 de diciembre de 2012.

Vallespín, F. (2012). La mentira os hará libres. Realidad y ficción en la democracia. Barcelona: Galaxia Gutenberg.

Vidal, M. (2010). «Por qué evitar la expresión "Software de Fuentes Abiertas"», en Novática, número 203, enero-febrero de 2010, pp. 67-69. Documento accesible en línea: <http://gsyc.es/ mvidal/docs/no-sfa.pdf>. Fecha de consulta: 29 de diciembre de 2012.

Winner, L. (2008). La ballena y el reactor. Una búsqueda de los límites en la era de la alta tecnología. Barcelona: Editorial Gedisa. 